\title{
Kosterlitz-Thouless-type caging-uncaging transition in a quasi-one-dimensional hard disk system
}

\author{
A. Huerta $\odot,{ }^{1}$ T. Bryk $\odot,{ }^{2,3}$ V. M. Pergamenshchik, ${ }^{4}$ and A. Trokhymchuk $\odot^{2}$ \\ ${ }^{1}$ Facultad de Física, Universidad Veracruzana, Circuito Gonzálo Aguirre Beltrán s/n Zona Universitaria, \\ Xalapa, Veracruz, C.P. 91000, México \\ ${ }^{2}$ Institute for Condensed Matter Physics, National Academy of Sciences of Ukraine, 1 Svientsitskii Street, UA-79011 Lviv, Ukraine \\ ${ }^{3}$ Institute of Applied Mathematics and Fundamental Sciences, Lviv Polytechnic National University, UA-79013 Lviv, Ukraine \\ ${ }^{4}$ Institute of Physics, prospect Nauki, 46, Kyiv 03039, Ukraine
}

(Received 18 March 2019; revised 9 April 2020; accepted 12 August 2020; published 2 September 2020)

\begin{abstract}
The simplicity of a quasi-one-dimensional system of hard disks enables us to get a much deeper and more quantitative insight into the solid-to-fluid transformation and identify it as caging-uncaging transition. Both computer simulation data and theoretical results show that density decrease induces progressively larger number of uncaged disk pairs which exchange their transverse positions through windows in the initial crystalline zigzag array. At low densities windows dominate and disks are moving independently (pairs "dissociate"). Along with the simulation data on the transversal excitation modes and pair correlation function this shows that the solid-toliquid transformation is a continuous Kosterlitz-Thouless-type transition.
\end{abstract}

DOI: 10.1103/PhysRevResearch.2.033351

\section{INTRODUCTION}

The interest in a quasi-one-dimensional (q1D) hard-core fluid has both basic [1-6] and applied [7-10] aspects. The fundamental interest is because this system allows for a more detailed [1] and more advanced analytical approach [11-14] which can facilitate studies in higher dimensions. The freezing-melting transition [15] and collective dynamics $[1,16]$ in 2D hard-core systems are related to the caging phenomenon [17], which is a hindrance of particle motion by its nearest neighbors (NN) [18]. We resort to the q1D hard disk (HD) system confined to a pore to get insight into the role of caging-uncaging events. Recently [19] one of us developed a quantitative analytical theory of the structural transformations in this system. Here we combine these theoretical predictions with the molecular dynamic (MD) simulation data [20].

The fundamental question related to the q1D HD system is as follows. A solid-to-fluid transition is a global phenomenon attributed to the entire body, but in a 2D crystals it starts from local emergence of bounded defect pairs [21]. This might have similarity to the melting in a q1D HD system. Its densely packed state is the zigzag array where all disks are caged: each disk touches just one wall and is prohibited from moving across the pore by two NNs. As density decreases, to gain entropy the system searches for uncaging. Though uncaging cannot occur in the entire system, it can occur locally where pair of disks tries to exchange their positions across the pore.

Published by the American Physical Society under the terms of the Creative Commons Attribution 4.0 International license. Further distribution of this work must maintain attribution to the author(s) and the published article's title, journal citation, and DOI.
Usually, however, the density of defects is determined by their core energy via Boltzmann's factor, which is irrelevant to HD systems where possible defects have a purely entropic origin. Can such entropy-driven local uncaging, resembling thermal excitation of bounded pairs in the Kosterlitz-Thouless scenario, be effective in the zigzag melting? Our findings support the effectiveness. As density decreases, both simulation and theory predict an emergence of progressively larger number of uncaged disk pairs. They first must be strongly correlated, and the pair correlations along the channel are indeed found to weaken as a power law. But eventually the uncaging dominates, transverse excitation modes signal that HDs can fly between the confining walls, and the correlations decay exponentially. This picture is in line with a continuous Kosterlitz-Thouless-type transition.

\section{QUASI-LONG-TO-SHORT-RANGE-ORDER TRANSFORMATION}

A q1D HD system is modeled by $N$ HDs of diameter $d$ in a rectangular pore formed by two horizontal hard walls of length $L$. The walls are separated in the $y$ direction by the width $D<2 d$ such that disks cannot pass each other (Fig. 1). We consider a q1D HD system of width $D=3 / 2 d$.

The disk-disk, $u$, and disk-wall, $u_{\mathrm{w}}$, interaction potentials are of the standard form: $u(r)=\infty$ for $r<d, u=0$ for $r \geqslant d ; u_{\mathrm{w}}(y)=0$ for $d / 2<y<d, u_{\mathrm{w}}=\infty$ otherwise, where $r=\left|\mathbf{r}_{\mathbf{i}}-\mathbf{r}_{j}\right|$ is the distance between disks $i$ and $j$. The periodic boundary conditions in the $x$ direction are introduced. We employed the event-driven MD simulation algorithm [22] in the canonical $N V T$ ensemble. Different densities $\rho=N d / L$ correspond to different $L$ for the same $N$. We studied 11 pore lengths, so that $\rho$ ranges from $\rho=0.5$ to 1.14 ; the close packing (cp) crystalline zigzag $\rho_{\mathrm{cp}}=1.1547$. Although the main body of MD simulations is for $N=200$, to examine 

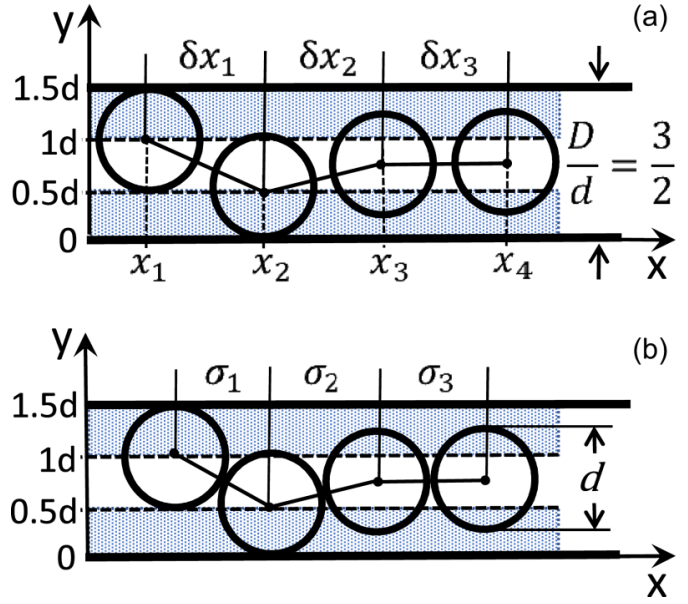

FIG. 1 . The pore of width $D=3 / 2 d$. (a) The $x$ distance between NN disks, $\delta x$. (b) The $x$ contact distance $\sigma$ for disks in (a). $\sigma_{1}=\sigma_{\mathrm{m}}, \sigma_{3}=d$; disk 4 can pass disk 3 in the $y$ direction.

size effects simulation runs with $N=20,400$, and 1000 were performed as well.

Figure 2 presents MD simulation data for the longitudinal static structure factor $S\left(k_{x}\right)$ and pair distribution function (pdf) $g(x)$. For the highest shown density $\rho=1.1111, S\left(k_{x}\right)$ exhibits the first and few neighboring peaks like a sheared-out $\delta$ function, pointing to a quasi-long-range order typical of a distorted crystal. As pore lengthens, the shape of $S\left(k_{x}\right)$ becomes typical of the short-range order in HD fluid. This is in accord with the reciprocal $2 \pi / k_{\max }$ of the position of the first peak of $S\left(k_{x}\right)$ versus per disk length $l=1 / \rho$ [inset in Fig. 2(a)]. We see a notable drift of $k_{\max }$ towards smaller values as $\rho$ decreases. In disordered systems, where the structural transition takes place, $k_{\max }(\rho)$ has different slopes on two sides of the transition [23]. The plot $2 \pi / k_{\max }$ versus $l$ is of this kind. For $\rho>1(l<d)$ where caging is essential, $2 \pi / k_{\max } \propto l$, which indicates that $k_{\max }$ can be just the vector of reciprocal lattice of the crystalline zigzag. At the same time, for densities $\rho<1,2 \pi / k_{\max }(l)$ deviates from the linear law as expected for disordered HD fluid.

The caging-dominated configuration at $\rho=1.1111$ is further confirmed in Fig. 2(b). Positions of the first and second peaks of $g(x)$ give information on the most probable distances between a chosen disk, its NN, and the next NN disks. For $\rho=1.1111$, the first and second peaks of $g(x)$ are located at $x / d \approx 0.880$ and 1.780 , respectively. This has to be compared with the case of the crystalline zigzag ordering when disks are fully caged (configuration snapshot for $\rho=1.111$; Fig. 2). Then the first peak should be at $x / d=\sqrt{3} / 2 \approx 0.866$, which is the minimum possible horizontal $\mathrm{NN}$ distance [minimum contact distance $\sigma_{\mathrm{m}}$; Fig. 1 (see below)], and the second peak at $x / d=2 \sigma_{\mathrm{m}}$. We see that for $\rho=1.1111$ the NN distance is nearly the same as that between two disks in the crystalline zigzag when they touch opposite walls (disks 1 and 2; Fig. 1). Moreover, that caging dominates in q1D HD system at $\rho=$ 1.1111 directly follows from the fact that the most probable next $\mathrm{NN}$ distance, $\sim 1.78 d$, is less than twice disk diameter, the gap for a disk is below $d$, and hence on average each disk is caged (see configuration snapshot for $\rho=1.1111$ ).
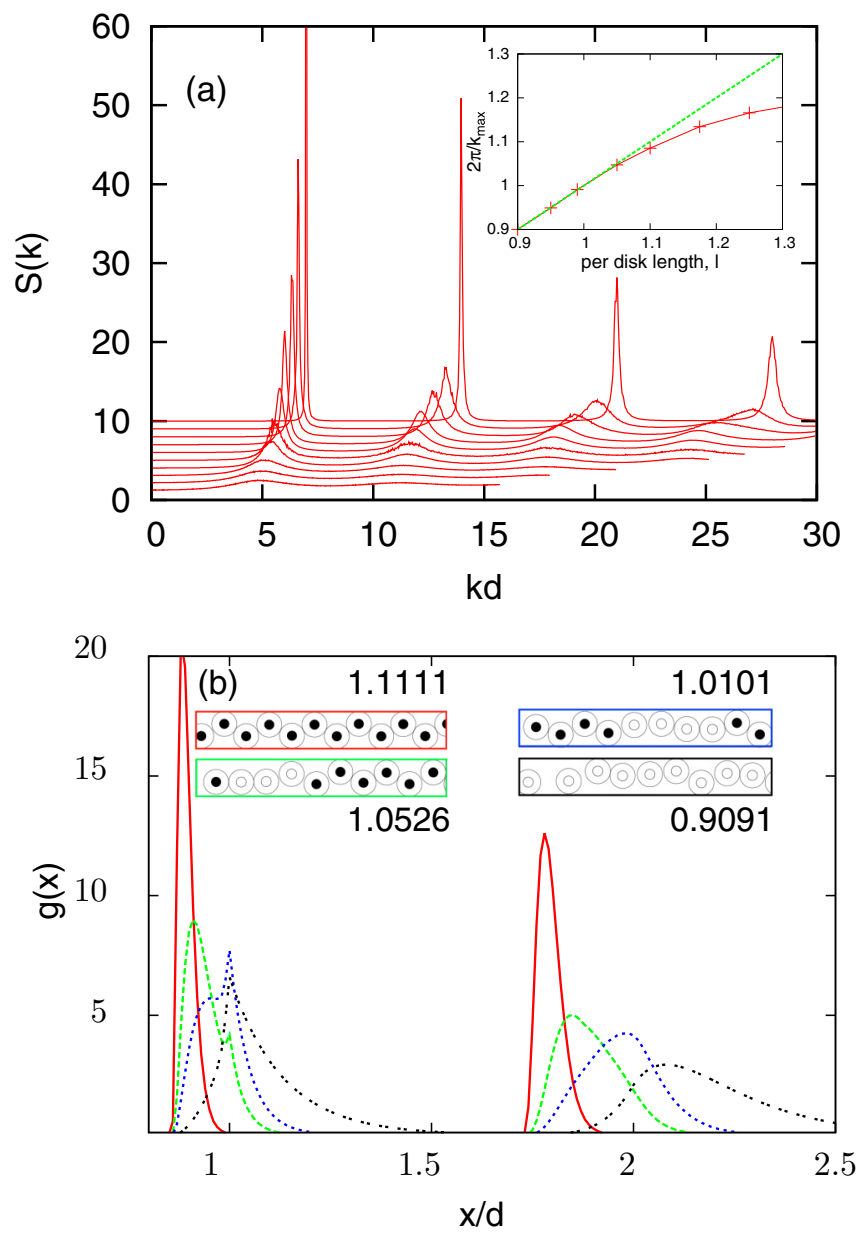

FIG. 2. (a) Structure factor $S\left(k_{x}\right)$ for 10 densities from $\rho=$ 0.1111 to 0.5 (in descending). Inset: Dependence $2 \pi / k_{\max }(l)$ for the first peak of $S\left(k_{x}\right)$. (b) Two peaks of $g(x)$ for $\rho=1.1111,1.0526$, 1.0101, and 0.9091 (from left to right) and configuration snapshots (inset). Filled circles indicate caged disks.

As density further decreases from $\rho=1.1111, g(x)$ shows developing two distinctive patterns. First, the peaks widen and their maxima positions shift to larger $x$, which is consistent with the increase of the per disk length $l$ and a more loose disk confinement. In addition, a sharp and narrow subpeak on the first peak is developing exactly at $x=d$. Below we show that this subpeak is related to and reflects an emergence of uncaging events in the zigzag array.

The MD data (Fig. 3) show that zigzag order is very different above and below some crossover density $\rho_{\mathrm{c}}$ from the interval between $1.056<\rho<1.1111$. Namely, we found that for $\rho=1.1111$, positional order decays as a power law $\sim x^{-2 / 3}$, whereas for $\rho=0.9091$ the decay is exponential, $\sim \exp [-(x / d) / 4]$. In spite of the linear dependence of $2 \pi / k_{\max }$ on $l$ for densities down to $\sim 0.95$ [inset in Fig. 2(a)], which apparently indicates a solidlike zigzag arrangement, the positional order for densities $\rho=1.0526$ and lower is already exponential. Thus, the above simulations data suggest that the quasi-long range at $\rho \gtrsim 1.1111$ somehow transforms into short-range order already for $\rho \sim 1.052$. The theory [19] shows that this is more a continuous crossover than a jumplike transition. 


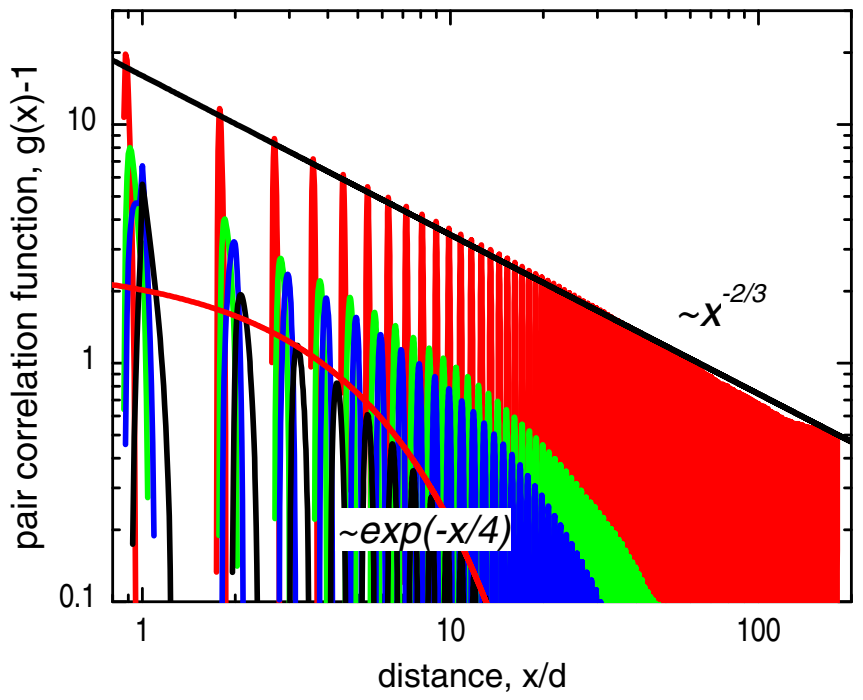

FIG. 3. Log-log plot of positional correlations $g(x)-1$ for system with $N=400$ at $\rho=1.1111,1.0526,1.0101$, and 0.9091 (from top to bottom). The solid straight and curved lines show a power $\sim x^{-2 / 3}$ and an exponential $\sim \exp [-(x / d) / 4]$ laws, respectively.

\section{SUBPEAK AND UNCAGING}

The key idea in the overall description of the structural transformation in a q1D HD system lies in the physics behind the narrow subpeak of $g(x)$ at $x=d$ developing below $\rho=$ 1.1111 [Fig. 2(b)]. Both theory [19] and simulations suggest that this subpeak is related to defects emerging in the zigzag array through which a pair of disks can exchange their vertical positions and get uncaged for some instants.

We introduce an important quantity $\sigma$ which is the $x$ projection of the contact distance between $\mathrm{NN}$ disks $[11,13,19]$. This $\sigma$ is the minimum possible horizontal separation of NNs for their coordinates $y_{1}$ and $y_{2}$ fixed [Fig. 1(b)]: $\sigma=$ $\sqrt{d^{2}-\left(y_{2}-y_{1}\right)^{2}}$.

It is clear that for $\rho \gtrsim 1$, when per disk length $l<1$, an exchange of disks' vertical coordinates can happen only locally, where the instant actual NN distance $\delta x=x_{2}-x_{1} \geqslant$ $d$. Both $\sigma$ and $\delta x$ depend on the difference $\delta y=y_{2}-y_{1}$ of their vertical coordinates (Fig. 1). As for $\rho \gtrsim 1, \sigma \approx \delta x$, distribution of $\sigma$ can give an accurate estimate for the number of windows with $\delta x \approx \sigma=d$. Hence $\delta x \approx \sigma_{m}=0.866 d$ is a zigzag pattern as then $\delta y=D-d=0.5 d$ and the NN disks are mutually caged, staying at the opposite walls. In contrast, $\delta x \gtrsim \sigma=d$ is a window pattern as then $\delta y \approx 0$ and the disks are uncaged and can traverse the pore. Pairs of contacting HDs with $\delta y \approx 0$ were earlier associated with defects in the study of glassy behavior of q1D HD system [1,5].

The distribution function $f_{\sigma}(\sigma)$ was obtained analytically [19] and for pore $D=3 / 2 d$ is shown in Fig. 4(a). As expected, for $\rho=1.14$ near the $\rho_{\mathrm{cp}}$ density, the zigzag pattern with $\sigma=\sigma_{\mathrm{m}}$ fully dominates. This pattern remains nearly the same until at $\rho=1.1111$ a very small maximum at the window pattern $\sigma=d$ appears and becomes very visible at $\rho=1.0526$. As $\rho$ further decreases, the fraction of $\sigma=\sigma_{\mathrm{m}}$ diminishes and that of $\sigma=d$ grows until it finally prevails at $\rho=0.5$. We conclude that a zigzag array, which is perfect
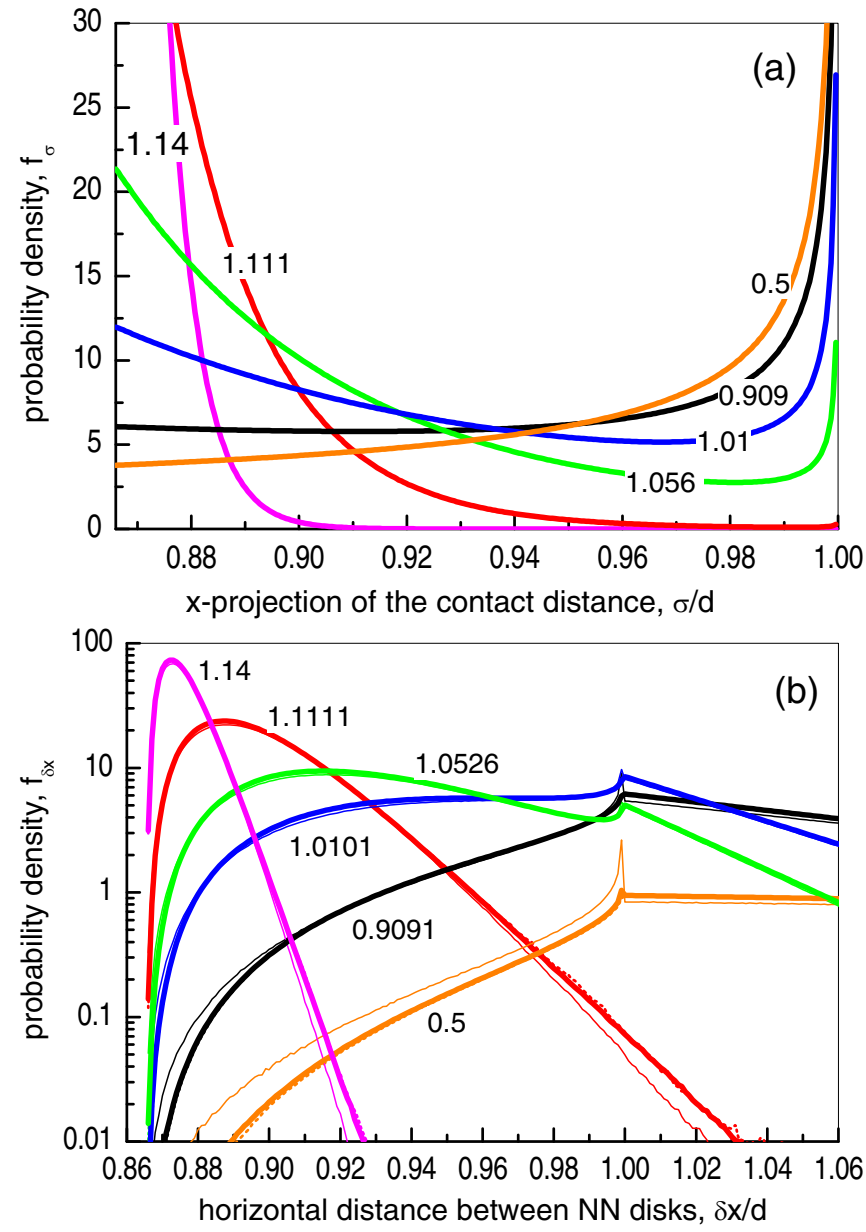

FIG. 4. (a) Prediction of theory [19]: distribution of $\sigma$ for the densities $\rho=0.5,0.909,1.01,1.056,1.111$, and 1.14 (from top to bottom when approaching $x / d=1$ ). (b) Semilog plot of MD distribution of $\delta x$ for $N=200$ and 1000 (indistinguishable, thick solid and thin dashed lines) and $N=20$ (thin solid lines) for the densities $\rho=1.14,1.1111,1.0526,1.0101,0.9091$, and 0.5 (from top to bottom when approaching $x / d=0.866$ ).

at $\rho_{\text {cp }}$, acquires windowlike defects whose number become substantial already for $\rho=1.0526$, continues to grow, and finally exceeds the zigzag pattern at fluid densities. Such an emergence of windows with $\sigma=d$ manifests itself as the subpeak on $g(x)$ at $x=d$ (Fig. 2). Note that this result is generic as the $\sigma$ distributions in the whole range of widths of a q1D HD single-file system are quite similar [19].

This picture is further confirmed by the distribution $f_{\delta x}$ of the actual distances $\delta x$ between NN disks obtained from simulations [Fig. 4(b)]. Similar to Fig. 4(a), for high $\rho=0.14$ and 0.1111 , these are distributed around $\delta x \sim 0.89 d$ close to $\sigma_{\mathrm{m}}$. However, at $\rho=1.0526$, in addition to a wide maximum at $\delta x \sim 0.92 d$, we see a sharp peak at $\delta x=d$. In contrast to the wide maximum drifting towards larger $\delta x$, the subpeak always remains at $\delta x=d$ although its shape is changing: it becomes more and more pronounced and finally exceeds the main peak signaling approaching to a liquidlike state. It points to the exclusive role that $\mathrm{NN}$ disk separation at $\delta x=d$ plays. Namely, creation of windows of the width of disk diameter $d$ in the zigzag array, neither wider nor narrower, is the most 
effective way to gain entropy by uncaging two disks and making them extend their wondering to the whole pore width; wider windows require a denser parent zigzag residue with lower entropy while narrower ones provide no uncaging.

\section{CAGING-UNCAGING AND TRANSVERSE EXCITATIONS}

In a 1D HD system, the vertical motion is absent. In a densely packed q1D HD system it is also prevented by the full caging, but it eventually appears as the confinement weakens. As for sufficiently low $\rho$ the vertical disks' motion from one wall to another is possible, here one expects a nearly ballistic transverse $(\mathrm{T})$ oscillation. As it comes from the maximum vertical path $D-d$, its frequency $\omega_{t 1}$ must be lowest possible. At high $\rho$, the $\sigma=d$ windows are rare and disks can bounce at most between one wall and the midplane, hence the lowest $\mathrm{T}$ frequency is $\omega_{t 2} \sim 2 \omega_{t 1}$. This $\omega_{t 2}$ is related to the maximal $\sigma$ at window nuclei which require local compression and must result in high-frequency longitudinal (L) and T jitters. One can thus expect that the lowest T frequency $\omega_{t 2}$ and the highest $\mathrm{L}$ and $\mathrm{T}$ frequencies appear near the same $k$. In addition, the group velocity at this $k$ range has to be zero as windows are not transferred by the waves. The dispersion $\omega(k)$ of the $\mathrm{L}$ and T excitations in a q1D HD system obtained from MD is in line with this picture (Fig. 5). For $\rho=1.0101$, when the order is of a short range, $\omega_{t 1}$ can be identified with the practically $k$ independent $\mathrm{T}$ frequency $\sim 10$ [Fig. 5(a)]. At the same time, for $\rho=1.1111$ when there are almost no $\sigma=d$ windows, the $\mathrm{T}$ spectrum splits into the lowest $\omega_{t 2} \sim 20 \approx 2 \omega_{t 1}$ and highest frequency $\omega_{t 3} \sim 100$ at those $k$ where $\mathrm{L}$ frequency is maximum [Fig. 5(b)]. The curves $\omega_{t 1}(k)$ and $\omega_{t 2}(k)$ are plateaus which indicate zero group velocities. The continuous $\mathrm{L}$ and $\mathrm{T}$ modes for $\rho=1.1111$ are related to the short free path oscillation of mutually caged disks near the walls. Thus, the main properties of $\omega_{t}(k)$, which is directly related to the vertical motion, confirm the role of the number of $\sigma=d$ windows in the zigzag arrangement.

\section{CONCLUSION}

In this paper we studied structural transformations in q1D HD array and found a strong similarity to a continuous temperature-driven Kosterlitz-Thouless-type transition from a solidlike to liquidlike state in $2 \mathrm{D}$ crystals. At $\rho_{c p}$ the disks form perfect zigzag. As confinement weakens, the tendency to the entropy increase results in an emergence of progressively larger number of windowlike defects through which pairs of NN disks uncage and exchange their vertical positions. In doing so the disks' motion within pairs is strongly correlated. The positional order in such a zigzag array with rare defects is
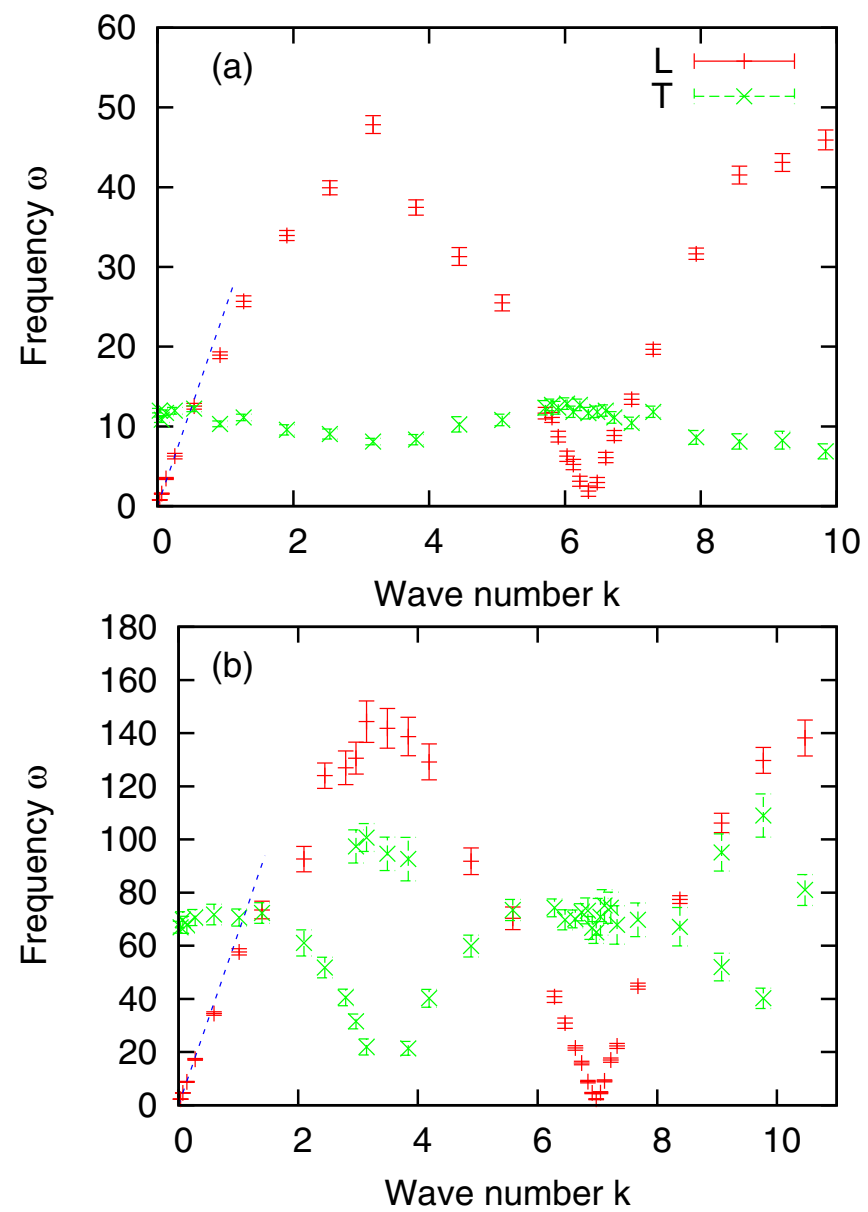

FIG. 5. Dispersion $\omega(k)$ of transverse (T) and longitudinal (L) excitations. (a) $\rho=1.0101$, (b) $\rho=1.1111$. Dashed line shows hydrodynamic dispersion.

quasi-long-range and decays as a power law. For even weaker confinement, the windows interconnect, disks can traverse the pore independently, and the pairs dissociate. Then the positional order is of short range, correlations decay exponentially, and the state is fluid. The cause for described path to the entropy increase in a q1D HD system is particularly strong and obvious: the dense caged system can gain entropy only via localized uncaging events, which results in the KosterlitzThouless-type scenario. The element specific for q1D HD system is that the windowlike defects in the zigzag array emerge via uncaging events.

\section{ACKNOWLEDGMENTS}

A.H. thanks Conacyt México for support during the sabbatical leave and Dra. Karen Volke and Dr. Alejandro Vasquez of the IFUNAM for valuable discussions. The work of V.M.P. was supported by VC 202 from National Academy of Sciences of Ukraine.
[1] C. L. Hicks, M. J. Wheatley, M. J. Godfrey, and M. A. Moore, Phys. Rev. Lett. 120, 225501 (2018).
[2] Y. Hu, L. Fu, and P. Charbonneau, Mol. Phys. 116, 3345 (2018). 
[3] L. Fu, C. Bian, C. W. Shields, D. F. Cruz, G. P. López, and P. Charbonneau, Soft Matter 13, 3296 (2017).

[4] K. Nygård, S. Sarman, K. Hyltegren, S. Chodankar, E. Perret, J. Buitenhuis, J. F. van der Veen, and R. Kjellander, Phys. Rev. X 6, 011014 (2016).

[5] J. F. Robinson, M. J. Godfrey, and M. A. Moore, Phys. Rev. E 93, 032101 (2016).

[6] M. Z. Yamchi, S. S. Ashwin, and R. K. Bowles, Phys. Rev. E 91, 022301 (2015).

[7] E. Fois, A. Gamba, G. Tabacchi, S. Quartieric, and G. Vezzalini, Phys. Chem. Chem. Phys. 3, 4158 (2001).

[8] J. Kofinger, G. Hummer, and C. Dellago, Phys. Chem. Chem. Phys. 13, 15403 (2011).

[9] A. Waghe, J. C. Rasaiah, and G. Hummer, J. Chem. Phys. 137, 044709 (2012).

[10] D. Mark, S. Haeberle, G. Roth, F. von Stetten, and R. Zengerle, Chem. Soc. Rev. 39, 1153 (2010).

[11] L. Tonks, Phys. Rev. 50, 955 (1936).

[12] J. A. Barker, Aust. J. Phys. 15, 127 (1962).
[13] D. A. Kofke and A. J. Post, J. Chem. Phys. 98, 4853 (1993).

[14] S. Varga, G. Balló, and P. Gurin, J. Stat. Mech. (2011) P11006.

[15] A. Huerta, D. Henderson, and A. Trokhymchuk, Phys. Rev. E 74, 061106 (2006).

[16] A. Huerta, T. Bryk, and A. Trokhymchuk, J. Colloid Interface Sci. 449, 357 (2015)

[17] T. M. Truskett, S. Torquato, S. Sastry, P. G. Debenedetti, and F. H. Stillinger, Phys. Rev. E 58, 3083 (1998).

[18] M. Goldstein, J. Chem. Phys. 51, 3728 (1969).

[19] V. M. Pergamenshchik, arXiv:2003.10714 (2020).

[20] A. Huerta, T. Bryk, and A. Trokhymchuk, arXiv:1904.05970 (2019).

[21] J. M. Kosterlitz and D. J. Thouless, J. Phys. C 6, 1181 (1973).

[22] M. P. Allen and D. J. Tildesley, Computer Simulation of Liquids (Science Publication, Oxford, 1989).

[23] F. A. Gorelli, S. De Panfilis, T. Bryk, L. Ulivi, G. Garbarino, P. Parisiades, and M. Santoro, J. Phys. Chem. Lett. 9, 2909 (2018). 